\title{
SUPERLUMINAL MOTIONS AND LARGE SCALE STRUCTURE IN 3C 120
}

\author{
R. C. Walker ${ }^{1}$, J. M. Benson ${ }^{2}$, and S. C. Unwin ${ }^{3}$ \\ ${ }^{1}$ National Radio Astronomy Observatory*, Socorro, N.M., U.S.A. \\ ${ }^{2}$ National Radio Astronomy Observatory*, Charlottesville, VA, U.S.A. \\ ${ }^{3}$ California Institute of Technology, Pasadena, CA, U.S.A.
}

\begin{abstract}
The jet in the superluminal source $3 \mathrm{C} 120$ has been observed on all scales from under a parsec to about $100 \mathrm{kpc}$. The jet brightness decays rather smoothly with core distance, indicating that the basic characteristics of the jet are established on parsec or smaller scales and evolve simply with distance.
\end{abstract}

3C 120 is the closest of the superluminal sources and has the highest angular expansion rates. It is associated with a galaxy that is usually classified as a Seyfert, although it is highly disturbed by the nuclear source and its morphological type is not clear. (cf. Baldwin et al. 1980). The parsec scale structure of 3C 120 has been monitored extensively since the early 1970's (Walker et al. 1982 and references therein). Clear epochs of expansion were seen in 1972-1974 and in 1979 with angular rates of about 1.5 mas per year. Between 1980 and 1983, four distinct knots were seen to emerge from the core and move away at a rate of about $2.5 \mathrm{mas} \mathrm{yr}^{-1}$ (about $4 c$ for $\mathrm{H}_{o}=100 \mathrm{~km} \mathrm{~s}^{-1} \mathrm{Mpc}^{-1}$ and a redshift of 0.033 ). The knots were resolved from the core at about 1 mas and decayed as they moved out until, at roughly 6 mas, limited dynamic range made them hard to follow. Some details of these motions can be found in Walker, Benson, and Unwin (1987).

Extensive VLBI and VLA observations of the radio structure of $3 \mathrm{C} 120$ on scales larger than that observed in the VLBI monitoring runs have been made (Walker, Benson, and Unwin 1987). They show that there is a continuous jet structure from the parsec scales, where the superluminal motions are seen, to over $100 \mathrm{kpc}$, well outside of the optical galaxy. In addition, diffuse structures are seen with an overall extent of at least $400 \mathrm{kpc}$ in projection. The jet is one sided and has a longitudinal magnetic field, characteristics typical of the more powerful radio sources (Bridle and Perley 1984). On the side opposite the jet, at about $60 \mathrm{kpc}$, there is an edge-brightened lobe. Any counterjet that feeds this lobe is at least 100 times weaker in the radio than the observed jet. The larger scale structures are very confused. This may be the result of the projection effects that are expected in the line-of-sight models for superluminal motions.

Perhaps the most significant result of the observations of the structure of $3 \mathrm{C} 120$ is the simple evolution of brightness that was found. Figure 1 shows the brightness as a function of jet width. It covers about 3.5 orders of magnitude in core distance and 8 orders of magnitude in brightness. The data are fit well by a power law. The simple evolution of the jet suggests that its basic character is established on parsec scales and it evolves simply, perhaps in response to a gradient of external pressure, as it travels out. The source was actually observed over 5 orders of magnitude in core distance and the simple evolution seems to hold over the full range. However the jet width on small

* The National Radio Astronomy Observatory is operated by Associated Universities Incorporated under contract to the National Science Foundation. 


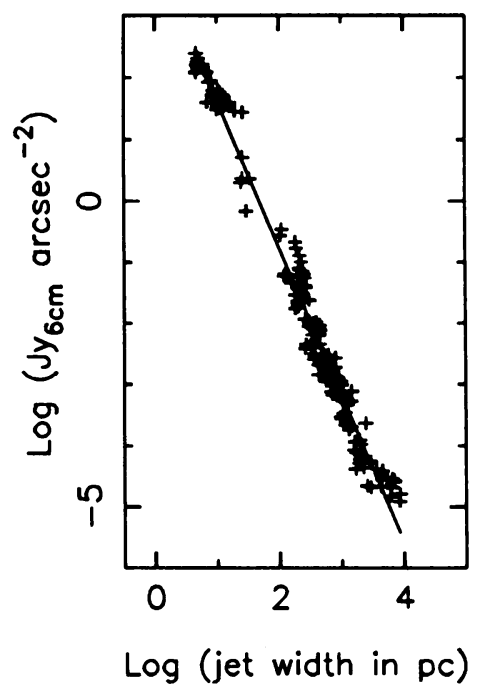

Figure 1: The brightness (Jy per square arcsecond) of the $3 \mathrm{C} 120$ jet vs. the width of the jet in parsecs. The solid line represents a power law with an index of $-2.40 \pm 0.08$ that is the result of a least squares fit to the data.

scales and the brightness on large scales could not be measured reliably so the full range is not used in Figure 1.

With the brightness information shown in Figure 1, the physical parameters of the jet can be calculated under the assumption that there is equipartition between the energy in relativistic particles and magnetic field. Some results are:

Minimum energy density: $U_{\min }=1.5 \times 10^{-10} r^{-1.95} \mathrm{erg} \mathrm{cm}^{-3}$

Magnetic field: $B=4.1 \times 10^{-5} r^{-0.97}$ gauss

Pressure: $p / k=3.7 \times 10^{5} r^{-1.95} \mathrm{~cm}^{-3} \mathrm{deg}$,

where the pressure is given in units of density times temperature, and $r$ is the FWHM of the jet in arc seconds.

The pressure is about what is expected for the external medium on kpc and larger scales but is somewhat lower that what is expected in the broad and narrow line regions on small scales. The evolution of brightness is not consistent with a flux conserving, adiabatic expansion of a jet with a longitudinal field even with slowing of the bulk velocity (Bridle and Perley 1984) - the velocity would become too low to reach the outer structures. The data appear to be most consistent with a light jet, containing little or no thermal material, that is relativistic at least on small scales and perhaps on all scales. For further details, see Walker, Benson, and Unwin (1987).

\section{REFERENCES}

Baldwin, J. A., Carswell, R. F., Wampler, E. J., Smith, H. E., Burbidge, E. M., and Boksenberg, A. 1980, Ap. J., 236, 388.

Bridle, A. H., and Perley, R. A. 1984, Ann. Rev. Astron. Ap., 22, 319.

Walker, R. C., Benson, J. M., and Unwin, S. C. 1987, Ap. J., 316, 546.

Walker, R. C., Benson, J. M., and Unwin, S. C. 1987, in Superluminal Radio Sources, ed. J. A. Zensus and T. J. Pearson (Cambridge: Cambridge University Press), p. 48. 